\title{
Specimen Fracturing Device for Ion Microprobe Mass Analyzer and its Application to the Analysis of Grain Boundary Segregation
}

\author{
By Kimitaka Sato*, Ken'ichi Suzuki*, Ryutaro Matsumoto* \\ and Shin'ichi Nagashima*
}

\begin{abstract}
Although a great deal of work has been performed on the dependence of strength and toughness on grain size in steels, the grain boundary properties in connection with strength and toughness remain unclarified. In order to study the grain boundary properties, the specimen fracturing device developed in the authors' laboratory was incorporated in the ion microprobe mass analyzer. Utilizing this device, specimens can be fractured mechanically in high vacuum and the distribution and segregation of various elements at grain boundaries can be assessed quantitatively. The practical application of this device to the study of grain boundary segregation of $\mathrm{Fe}-1.5 \% \mathrm{Sn}$ and $\mathrm{Fe}-3 \% \mathrm{Mn}-0.4 \% \mathrm{Mo}$ steels is described.
\end{abstract}

(Received May 31, 1976)

\section{Introduction}

In the metallurgical study, especially, it is very important to clarify the segregating and concentrating conditions of some specific elements in the grain boundaries for the investigation of fundamental properties, such as temper brittleness ${ }^{(1)}$. Since the segregation of the grain boundaries are very small in width, usually several tens of Angströms, applicable analyzing techniques are limited. Among the applicable techniques, the ion microprobe mass analyzer (IMA) is expected to be used effectively. The analysis of the grain boundaries by the IMA may be effected according to the following two methods:

(1) Line analysis method by use of the secondary ion image ${ }^{(2)(3)}$.

This method will give very valuable knowledge if the distribution of elements segregating in the grain boundaries would be really observed, but is not practicable in many cases owing to the limitations of the beam diameter (i.e. the resolving power) of the primary ion.

(2) In-depth analysis of fractured surface. If the specimen is fractured under the same vacuum as prevailing in the analyzer, con-

* Fundamental Research Laboratories, Nippon Steel Corporation, Ida, Nakahara-ku, Kawasaki 211, Japan. tamination of its surface can be prevented and the fractured specimen can immediately be put to analysis; therefore, the distribution of elements segregating in the grain boundaries can be observed by in-depth analysis.

To make use of the advantage of the second method, a fracturing device attachable to the IMA has been developed. This device is designed to fracture the specimen under the same vacuum as is present in the IMA and to analyze the fracture in a condition in which it will not be contaminated nor oxidized. In designing the device, the present authors made the most of their experience in the development of a similar kind of device adapted to be attached to the Auger electron spectrometer ${ }^{(4)}$.

\section{Experimental}

\section{Ion microprobe mass $\operatorname{analyzer}^{(5) \sim(7)}$}

In order to meet the experimental requirements, a modified Hitachi model IMA-2 ion microanalyzer was chosen. The design of the IMA was discussed before its construction. An ion pump with the capacity of $400 \ell / \mathrm{sec}$ is employed for the evacuation of the analyzer, while part of ion gun is evacuated with an oil diffusion pump.

In order to study the effect of the impurity ions upon analytical results, a special accessory was designed. It has a function such that the 
primary ion can be separated before reaching the sample surfaces and, therefore, the comparison can be made of between the results before and after removal of impurity ions. Liebl $^{(8)}$ used a magnetic mass spectrometer for this purpose, but the device has large size and is difficult to measure in the presence of impurity ions because of the non-linear beam. As the device for the separation of primary ion, a

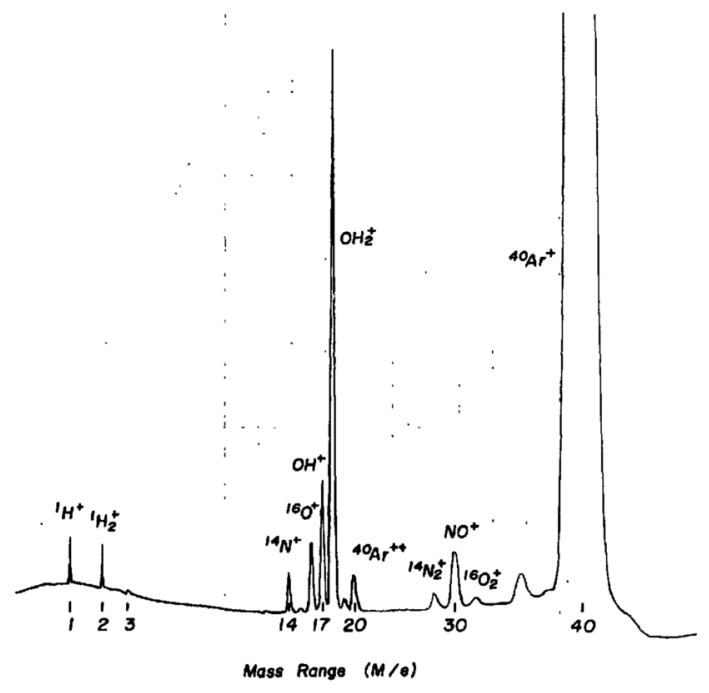

Fig. 1 Mass spectrum of primary argon ion beam just after setting an ion pump (400 $\ell / \mathrm{sec})$.

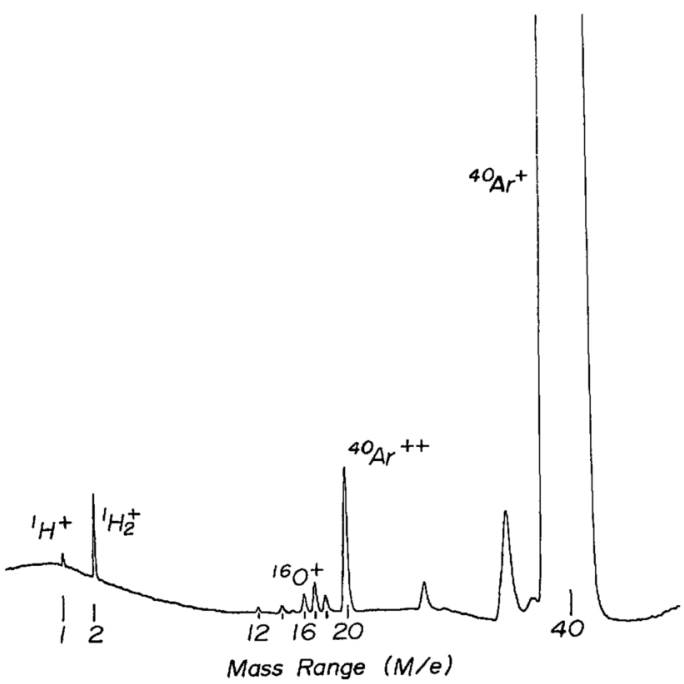

Fig. 2 Mass spectrum of primary argon ion beam after passing 50 days from setting an ion pump $(400 \ell / \mathrm{sec})$.
Wien filter ${ }^{(6)(9)}$ was installed because of its small size, superior permeability and linear structure. Figures 1 and 2 show the mass spectrum of primary ion without separation obtained by a magnetic scanning of the Wien filter. Impurity ions can be reasonably separated by setting the magnetic field of the filter in the mass range of ${ }^{40} \mathrm{Ar}^{+}$.

\section{Specimen fracturing device}

This new specimen-fracturing device is fitted to the specimen holder-introducing section of the IMA. The fractured specimen is delivered to the specimen holder-introducing section as it is held in the specimen holder, and thence to the specimen chamber under vacuum according to the ordinary specimen-introducing procedure.

This specimen-fracturing device mainly consists of a specimen holder-introducing section, a specimen-fracturing chamber and a specimenholder transfer handle, plus an observation window, a specimen-cooling section and a thermocouple for measuring the temperature of the specimen to be fractured. Figure 3 is a cross-sectional view of the principal portion of the device. The specimen to be fractured, as a general rule, is a round rod $20 \mathrm{~mm}$ long and 4 $\mathrm{mm}$ in diameter, having a $\mathrm{v}$-shaped notch of $0.5 \mathrm{~mm}$ depth at the middle. Four specimens are set in the holder at a time, and fractured one

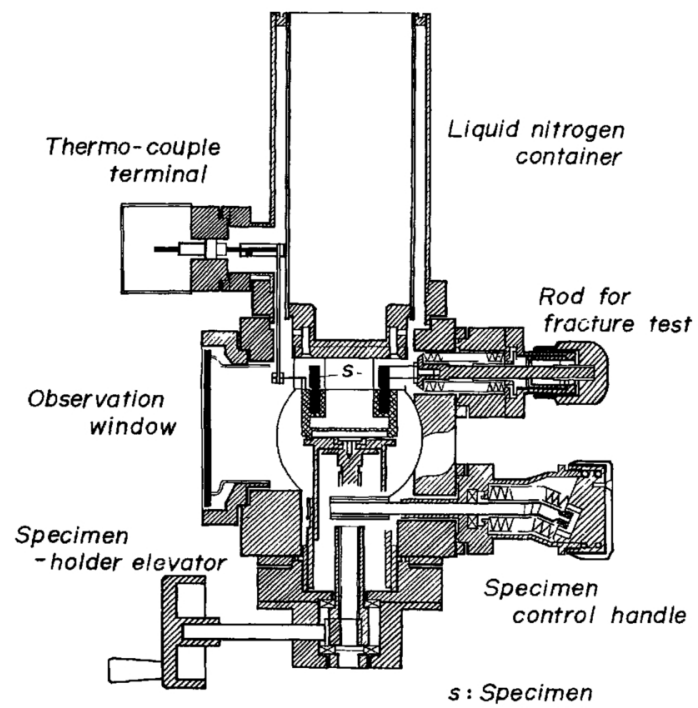

Fig. 3 Cross section of specimen fracturing device. 
after another by lightly hitting with a hammer.

\section{Results and Discussion}

\section{Features of the specimen fracturing device}

Before fracturing, the specimen is cooled to $-160^{\circ} \mathrm{C}$ by use of liquid nitrogen. The cooling condition is monitored by means of a recorder on which signals from the thermocouple are displayed as illustrated in Fig. 4. The specimen is usually cooled to $-160^{\circ} \mathrm{C}$ in about $10 \mathrm{~min}$ when it is cooled for the first time. The four steps in the cooling-characteristics curve of Fig. 4 is due to the rotation of the specimen holder through an angle of 90 degrees when each of the four specimens is fractured, thereby the holder being temporarily displaced out of the measuring position of the thermocouple.

In order to confirm the effect of fracturing the specimen in vacuo, specimens of $\mathrm{Fe}-1.5 \% \mathrm{Sn}$ alloy were fractured under vacuum and then divided into two groups. The first group of the fractured specimens was immediately analyzed under the same vacuum, while the second group was taken out of vacuum, allowed to expose to the atmosphere for an hour, and then returned into the IMA for analysis. The conditions of contamination and adsorption at the fracture of the specimens of these two groups were compared as shown in Figs. 5 and 6. In the specimens exposed to the atmosphere, the ion intensities of ${ }^{12} \mathrm{C}^{+}$and ${ }^{16} \mathrm{O}^{+}$indicated an interesting change in the direction of depth; that is, carbon was present more in the uppermost surface of the specimen than in any other portions, and oxygen increased with decreasing

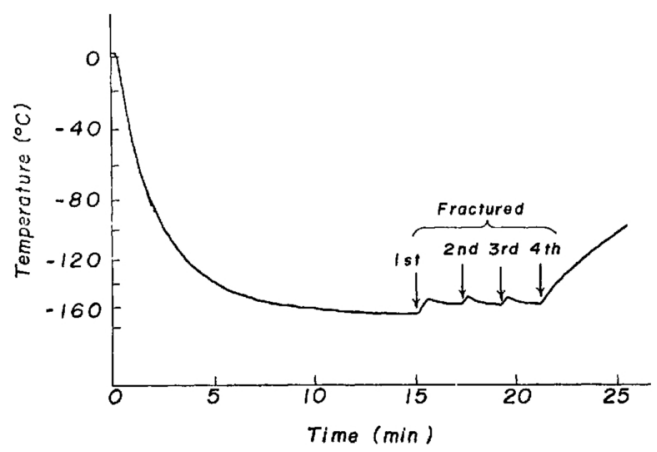

Fig. 4 Characteristic colling curve of specimen in the specimen fracturing device.

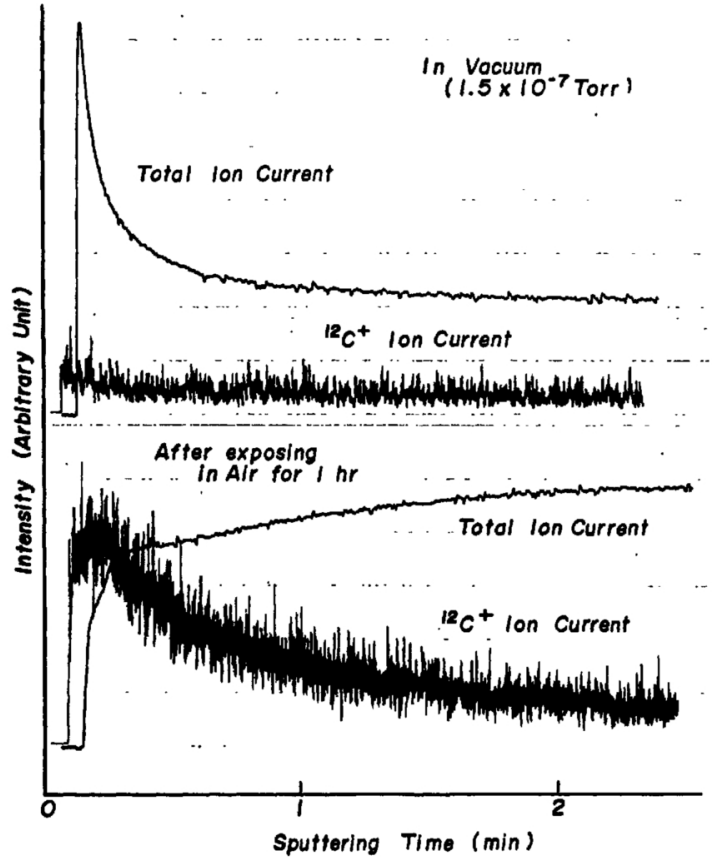

Fig. 5 In-depth distribution of carbon on fractured surface in vacuo and after exposing in air.

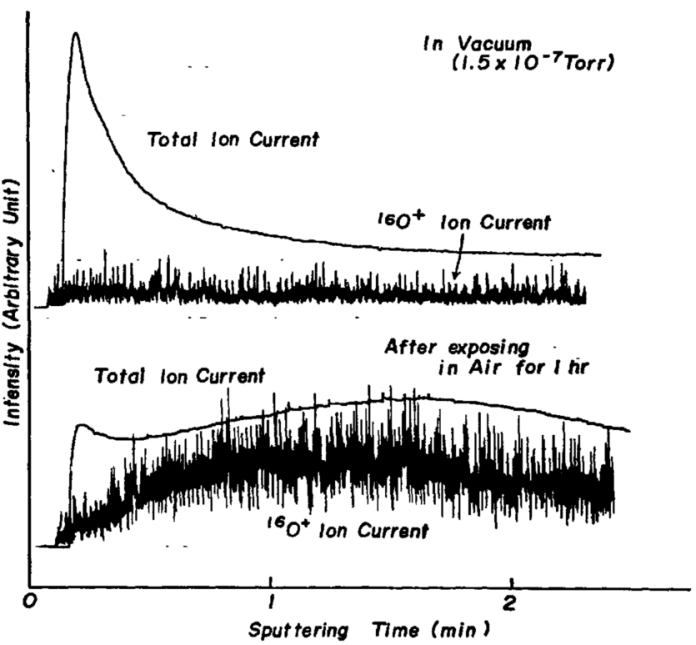

Fig. 6 In-depth distribution of oxygen on fractured surface in vacuo and after exposing in air.

carbon, progressing inward from surface to center of the specimen. On the other hand, the specimens subjected to immediate analysis under vacuum did not indicate the presence of carbon or oxygen in their surface. These data were obtained at the best sensitivity of the IMA. The aforesaid phenomenon apparently shows 
that the fracturing of specimens under vacuum produces a remarkably excellent effect. Particularly, it is understood that a very thin film of oxide is formed at the fracture of the specimen once it is exposed to the atmosphere, and carbon is adsorbed on the surface of the film.

\section{Analysis of grain boundary segregation}

Now some examples will be introduced in which the fracture in the grain boundaries was analyzed by use of the specimen fracturing device under discussion.

A first specimen was prepared by adding $1.5 \% \mathrm{Sn}$ to electrolytic iron, which was held at $1250^{\circ} \mathrm{C}$ for an hour and then quenched in iced salt water. The concentration of tin along the grain boundaries of this specimen had pre-

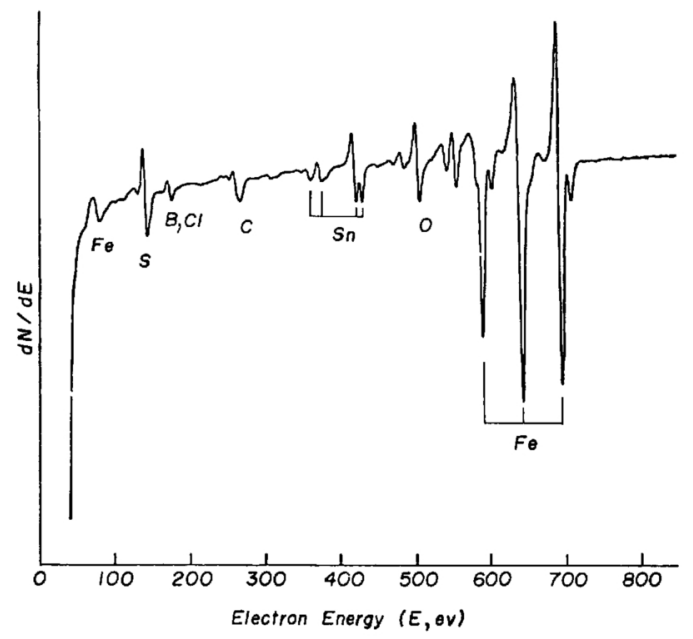

Fig. 7 An Auger electron spectrum of the fractured surface of $\mathrm{Fe}-1.5 \% \mathrm{Sn}$ alloy.



Fig. 8 In-depth distribution of tin in the fractured surface of $\mathrm{Fe}-1.5 \% \mathrm{Sn}$ alloy. viously been ascertained by the Auger electron spectrometry. An example of the Auger electron spectrum at the upper-most surface of this specimen is shown in Fig. 7. The IMA analysis was conducted under a condition wherein the diameter of the primary ion beam was enlarged to $700 \mu \mathrm{m}$ and the specimen current was built at $3 \mathrm{nA}$, so that the current density should be held as low as possible. This specimen was examined for the concentration distribution of ${ }^{120} \mathrm{Sn}^{+}$from the fracture surface to the center of the specimen. For this purpose, a change in total ion current was detected and measured simultaneously with a change in ion intensity with the passage of time, which was taken as a monitor to correct the ion intensity ${ }^{(10)}$. The curve as shown in Fig. 8 was obtained thereby. Further, the concentration of tin in the grain boundaries became apparent as illustrated in Fig. 9 even if the ion intensity of ${ }^{120} \mathrm{Sn}^{+}$and ${ }^{56} \mathrm{Fe}^{+}$was corrected by the total ion current.

A second specimen consisted of a steel of the $3 \% \mathrm{Mn}-0.4 \% \mathrm{Mo}-0.1 \% \mathrm{C}$ type with additions of nickel and chromium, which was specially prepared for the purpose of the test and subjected to solid solution, toughening and embrittling treatments. This specimen was analyzed under the same condition as for the first specimen. The results of the analysis are as shown in Fig. 10. Namely, a tendency was observed wherein there were more molybdenum, nickel and copper and less manganese and chromium in the grain boundaries than in the grains.

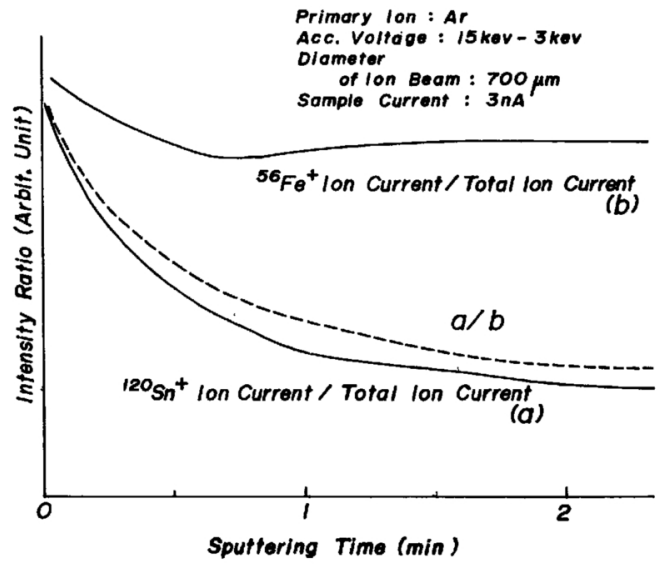

Fig. 9 Concentration of tin in the fractured surface of $\mathrm{Fe}-1.5 \% \mathrm{Sn}$ alloy (normalized curve). 

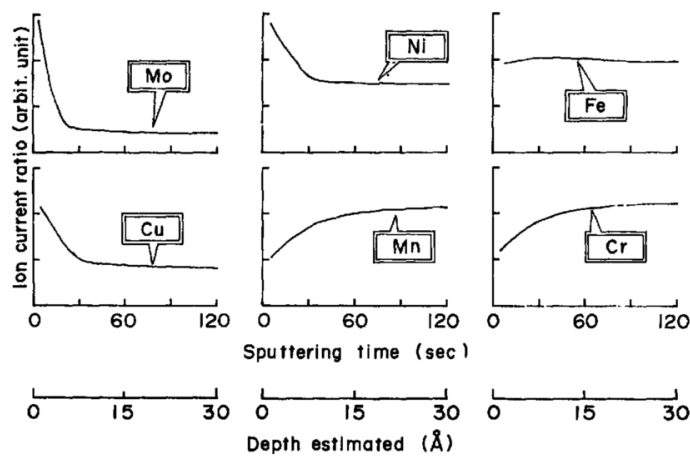

Fig. 10 Concentration of alloy elements in the grain boundary of $3 \% \mathrm{Mn}-0.4 \% \mathrm{Mo}-0.1 \% \mathrm{C}$ typed steel.

Judging from the sputtering rate, the aforesaid concentrated layer in the grain boundaries seems to be a monolayer or bilayer at the most.

\section{Conclusion}

As discussed above, the present specimen fracturing device permits us to analyze the specimen as fractured under vacuum without exposing it to the atmosphere. A careful use of this device will open up new ways, to the best advantage of IMA analysis, of gaining information about grain boundaries beyond the scope of capability of Auger electron spectroscopy and other analytical methods. Accordingly, much expectations may be entertained of future work on the device.

\section{Acknowledgments}

The authors wish to express their sincere thanks to Dr. N. Yamaguchi for his discussion on measuring and qualifying the analysis, and to Dr. M. Nagumo, Dr. T. Inoue and Mr. H. G. Suzuki for their encouragement and valuable discussions on temper embrittleness. They are also indebted to Dr. H. Tamura and Dr. Y. Nakajima of Hitachi Ltd. for their helpful discussions on instrumentation.

\section{REFERENCES}

(1) K. Sato and K. Tsunoyama: Oyo Buturi, 45 (1976), 828.

(2) J. M. Walsh and B. H. Kear: Met. Trans., 6A (1975), 226.

(3) $\mathrm{Ph}$. Maitrepierre, D. Thivellier and R. Tricot: Met. Trans., 6A (1975), 287.

(4) H. G. Suzuki and M. Ono: Trans. ISIJ, 12 (1972), 251.

(5) R. Matsumoto, K. Sato and K. Suzuki: Japan. J. Appl. Phys., Suppl. 2, Pt. 1 (1974), 387.

(6) K. Sato and H. Tamura: J. Vacuum Soc. Japan, 17 (1974), 385.

(7) R. Matsumoto, K. Sato and K. Suzuki: Tetsuto-Hagane (Japan), 60 (1974), 1980.

(8) H. Liebl: J. Appl. Phys., 38 (1967), 5277.

(9) T. Kondo, H. Tamura and H. Hirose: Mass Spectros. (Japan), 22 (1974), 229.

(10) H. Tamura, T. Kondo, I. Kanomata, K. Nakamura and Y. Nakajima: Japan. J. Appl. Phys., Suppl. 2, Pt. 1 (1974), 379. 